\title{
On the possible generation of the young massive open clusters Stephenson 2 and BDSB 122 by $\omega$ Centauri
}

\author{
G. M. Salerno ${ }^{1}$, E. Bica ${ }^{1}$, C. Bonatto ${ }^{1}$, and I. Rodrigues ${ }^{2}$ \\ 1 Universidade Federal do Rio Grande do Sul, Departamento de Astronomia CP 15051, RS, Porto Alegre 91501-970, Brazil \\ e-mail: [salerno; bica; charles]@if.ufrgs.br; irapuan@univap.br \\ 2 IP\&D - Universidade do Vale do Paraíba - UNIVAP, Av. Shishima Hifumi, 2911 - Urbanova, São José dos Campos 12244-000, \\ SP, Brazil
}

Received 27 January 2009 / Accepted 10 February 2009

ABSTRACT

\begin{abstract}
Context. Passing through the disk of a galaxy, a massive object such as a globular cluster can trigger star formation. Aims. We test the hypothesis that the most massive globular cluster in the Galaxy, $\omega$ Centauri, which crossed the disk approximately $24 \pm 2$ Myr ago, may have triggered the formation of the open clusters Stephenson 2 and BDSB 122.

Methods. The orbits of $\omega$ Centauri, Stephenson 2, and BDSB 122 are computed for the three-component model of Johnston, Hernquist $\&$ Bolte, which considers the disk, spheroidal, and halo gravitational potentials.

Results. With the reconstructed orbit of $\omega$ Centauri, we show that the latest impact site is consistent, within significant uncertainties, with the birth-site of the young massive open clusters BDSB 122 and Stephenson 2. Within the uncertainties, this scenario is consistent with the timescale of their backward motion in the disk, shock propagation and delayed star formation.

Conclusions. Together with open cluster formation associated with density waves in spiral arms, the present results are consistent with massive globular clusters being additional progenitors of open clusters, the most massive ones in particular.
\end{abstract}

Key words. galaxy: globular clusters: individual: $\omega$ Centauri - galaxy: open clusters and associations: individual: BDSB Galaxy: open clusters and associations: individual: Stephenson 2

\section{Introduction}

Disk-stability criteria and impact assumptions suggest that the passage of a globular cluster (GC) can trigger a bubble or wave of self-propagating star formation within the disk of the Galaxy (Wallin et al. 1996). The initial mechanical perturbation produces a local enhancement in the interstellar medium (ISM) density, from which localised star formation may occur. Subsequently, clustered star formation may happen along the border of a radially expanding density wave or ionisation front (e.g., Soria et al. 2005 - hereafter SCP05; Elmegreen \& Lada 1977; Whitworth et al. 1994). The expanding bubble is capable of compressing the neutral ISM above the stability criterion against gravitational collapse. Alternative star-formation triggering mechanisms are the infall of a high-velocity HI cloud (Elmegreen et al. 2000; Larsen et al. 2002), or hypernova explosions (Paczyński 1998).

Prominent, isolated star-forming bubbles have been observed in external galaxies. A bubble of diameter $\approx 600 \mathrm{pc}$ was detected in NGC 6946 (Larsen et al. 2002), containing a young super star cluster and at least 12 surrounding young clusters, the latter being comparable in luminosity to the most luminous Galactic OCs. The triggering mechanism in NGC 6946 appears to be the impact of a high-velocity HI cloud and/or hypernova explosions (Elmegreen et al. 2000). The Galaxy may harbour similar structures, a possible example being the Cygnus superbubble, which contains OB associations (Vlemmings et al. 2004, and references therein).

For a GC, the triggering effects are essentially gravitational. A natural assumption is that GCs, crossing the disk every $1 \mathrm{Myr}$ on average, may be responsible for some star formation. A possible case relates the origin of the OC NGC 6231 to the
GC NGC 6397 disk-crossing (Rees \& Cudworth 2003). Another possibility is that the low-mass GC FSR 584 has triggered star formation in the W 3 complex (Bica et al. 2007).

The OCs Stephenson 2 and BDSB 122 were discovered in 1990 (Stephenson 1990) and 2003 (Bica et al. 2003), respectively. $2 \mathrm{MASS}^{1}$ images of both clusters are shown in Fig. 1. The suspected richness of Stephenson 2 in red supergiants was confirmed by Nakaya et al. (2001) and Ortolani et al. (2002), providing an age of $\approx 20 \mathrm{Myr}$, and a distance from the Sun of $d_{\odot}=6 \mathrm{kpc}$ (Ortolani et al. 2002). Both clusters are among the most massive OCs known in the Galaxy. BDSB 122 has 14 red supergiants, is located at $d_{\odot}=5.8 \mathrm{kpc}$ from the Sun, and has an estimated mass of $2-4 \times 10^{4} M_{\odot}$, and an age of 7-12 Myr (Figer et al. 2006). Stephenson 2 has 26 red supergiants, is located at $d_{\odot}=5.8_{-0.8}^{+1.9} \mathrm{kpc}$ from the Sun, has an estimated mass of $4 \times 10^{4} M_{\odot}$, and an age of $12-17 \mathrm{Myr}$ (Davies et al. 2007). Their distances from the Sun are identical, within uncertainties, and their projected separation on the sky is $\approx 100 \mathrm{pc}$. The designation Stephenson 2 was originally assigned by Ortolani et al. (2002), and also adopted by Dias et al. (2002, and updates). Stephenson 2 and BDSB 122 are clearly in the red supergiant (RSG) phase (Bica et al. 1990). Davies et al. (2007) referred to these clusters as RSGC 1 and RSGC 2, respectively.

The positions (and uncertainties) of both clusters, together with $\omega$ Centauri (NGC 5139), are shown in Fig. 2 superimposed on a schematic view of the Milky Way (based on Momany et al. 2006; and Drimmel \& Spergel 2001). BDSB 122 and Stephenson 2 are slightly closer to the Galactic centre than the Scutum-Crux arm. Several other young clusters from the

${ }^{1}$ http://www.ipac.caltech.edu/2mass/releases/allsky 

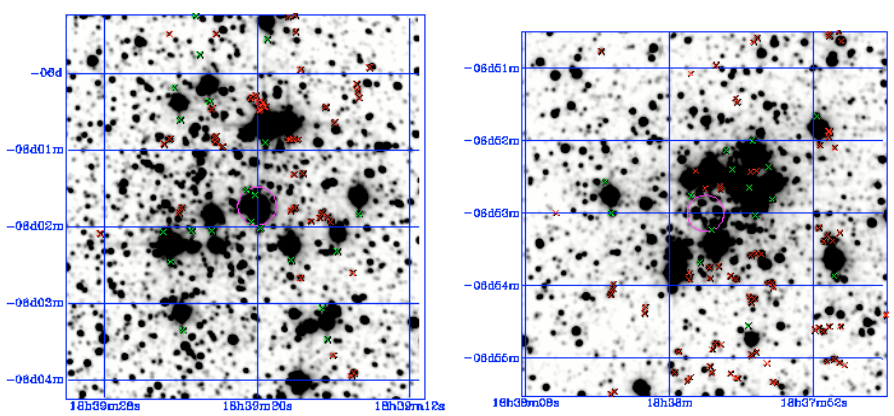

Fig. 1. $5^{\prime} \times 5^{\prime}$ 2MASS $K_{\mathrm{S}}$ images of Stephenson 2 (left) and BDSB 122 (right). Figure orientation: North to the top and East to the left.

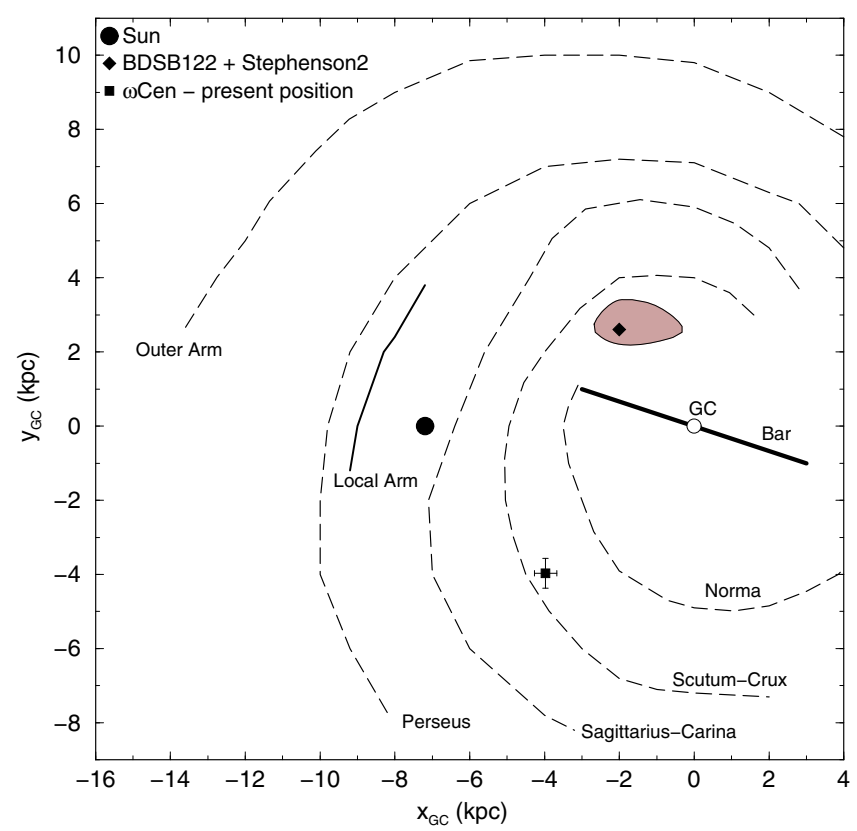

Fig. 2. The present-day positions (and uncertainties) of Stephenson 2, BDSB 122, and $\omega$ Centauri overplotted on a schematic projection of the Galaxy as seen from the North pole, with $7.2 \mathrm{kpc}$ as the Sun's distance to the Galactic centre. Main structures are identified.

catalogues of Bica et al. (2003) and Dutra et al. (2003) have already been studied in detail (e.g., Soares et al. 2008; Ortolani et al. 2008; Hanson \& Bubnick 2008).

We trace the orbits of both $\omega$ Centauri and Stephenson 2 backwards in time (and consequently, also that of BDSB 122) in the disk, testing an impact hypothesis for the origin of these two massive OCs. Using as constraints the GC space velocity, orbit integrations in the Galactic potential have been applied widely to 54 GCs (e.g., Dinescu et al. 2003; Allen et al. 2008).

This paper is structured as follows. In Sect. 2 we study the past orbit of $\omega$ Centauri. In Sect. 2.1, the past orbits of Stephenson 2 and $\omega$ Centauri are compared to search for spatial and time coincidence. Our conclusions are presented in Sect. 3.

\section{2. $\omega$ Centauri as a projectile}

The most massive Galactic GC, $\omega$ Centauri $\left(4 \times 10^{6} M_{\odot}\right.$ - Nakaya et al. 2001), has a metallicity spread and a flat density distribution typical of a dwarf galaxy nucleus captured by the Galaxy (Bekki \& Freeman 2003). Thus, irrespective of the existence of young massive clusters, in some way associated with the impact site, the orbit of $\omega$ Centauri in the Galactic potential
Table 1. Present-day cluster positions.

\begin{tabular}{lrrrr}
\hline \hline Cluster & $\ell$ & $b$ & $\alpha(J 2000)$ & $\delta(J 2000)$ \\
& $\left({ }^{\circ}\right)$ & $\left({ }^{\circ}\right)$ & $(\mathrm{h}: \mathrm{m}: \mathrm{s})$ & $\left({ }^{\circ},{ }^{\prime},{ }^{\prime \prime}\right)$ \\
$(1)$ & $(2)$ & $(3)$ & $(4)$ & $(5)$ \\
\hline$\omega$ Centauri & 309.10 & +14.97 & $13: 26: 46$ & $-47: 28: 37$ \\
BDSB 122 & 26.84 & +0.65 & $18: 37: 58$ & $-6: 53: 00$ \\
Stephenson 2 & 26.18 & -0.06 & $18: 39: 20$ & $-6: 01: 44$ \\
\hline
\end{tabular}

is worth consideration in searching, in particular, for the effects of the last disk passage. Evidence of a similar disk impact and a star-forming event has been observed in the spiral galaxy NGC 4559 with Hubble Space Telescope (HST), XMM-Newton, and ground-based (SCP05) data. The age of the star-forming complex, which has a ring-like distribution, is $\$ 30$ Myr. It appears to be an expanding wave of star formation, triggered by an initial density perturbation. The most likely triggering mechanism was a collision with a satellite dwarf galaxy crossing through the gas-rich outer disk of NGC 4559, which may have been the dwarf galaxy visible a few arcsec to the NW of the complex. This scenario is reminiscent of a scaled-down version of the Cartwheel galaxy (Struck-Marcell \& Higdon 1993; StruckMarcell et al. 1996).

As another example, proper motions (PMs) and radial velocity suggest that the GC NGC 6397 crossed the Galactic disk $5 \mathrm{Myr}$ ago, possibly triggering the formation of the OC NGC 6231 (Rees \& Cudworth 2003), and thus lending support to the present scenario (Wallin et al. 1996). NGC 6397 and NGC 6231 are closely projected on the sky $\left(\Delta \ell \approx 5^{\circ}, \Delta b \approx 13^{\circ}\right)$. However, in the case of the disk-crossing of $\omega$ Centauri being the possible triggering mechanism of BDSB 122 and Stephenson 2, the GC is now widely apart from the pair of massive OCs $\left(\Delta \ell \approx 77^{\circ}, \Delta b \approx 15^{\circ}\right.$ ). Thus, $\mathrm{PM}$ and radial velocity are fundamental constraints for the analysis of $\omega$ Centauri, and impact solutions require a detailed integration of its orbit across the Galactic potential.

\subsection{Orbit computation}

We employ a three-component mass-distribution model of the Galaxy resembling that in the study of a high-velocity black hole on a Galactic-halo orbit in the solar neighbourhood (Mirabel et al. 2001, and references therein). In short, we use the threecomponent model of Johnston et al. (1996) - hereafter JHB96 in which the disk, spheroidal, and halo gravitational potentials are described by $\phi_{\text {disk }}(R, z)=-G M_{\text {disk }} / \sqrt{R^{2}+\left(a+\sqrt{z^{2}+b^{2}}\right)^{2}}$ (Miyamoto \& Nagai 1975), $\phi_{\text {spher }}(R)=-\frac{G M_{\text {spher }}}{R+c}$ (Hernquist $1990)$, and $\phi_{\text {halo }}(R)=v_{\text {halo }}^{2} \ln \left(R^{2}+d^{2}\right)$, where $M_{\text {disk }}=1 \times$ $10^{11} M_{\odot}, M_{\text {spher }}=3.4 \times 10^{10} M_{\odot}, v_{\text {halo }}=128 \mathrm{~km} \mathrm{~s}^{-1}, R$ and $z$ are the cylindrical coordinates, and the scale lengths $a=$ $6.5 \mathrm{kpc}, b=0.26 \mathrm{kpc}, c=0.7 \mathrm{kpc}$, and $d=12.0 \mathrm{kpc}$. Table 1 shows the Galactic and Equatorial coordinates of the three clusters. Following Mizutani et al. (2003), the relevant parameters for computing the motion of $\omega$ Centauri are the distance from the Sun $d_{\odot}=5.3 \pm 0.5 \mathrm{kpc}$, the PM components $\left(\mathrm{mas} \mathrm{yr}^{-1}\right)$ $\mu_{\alpha} \cos (\delta)=-5.08 \pm 0.35$ and $\mu_{\delta}=-3.57 \pm 0.34$, and finally the heliocentric radial velocity $V_{r}=232.5 \pm 0.7 \mathrm{~km} \mathrm{~s}^{-1}$.

The models were computed with $R_{\mathrm{GC}}=7.2 \mathrm{kpc}$ (Bica et al. 2006) as the distance of the Sun to the Galactic centre. The Galactic velocities of $\omega$ Centauri are $U=54.3 \pm 9.5 \mathrm{~km} \mathrm{~s}^{-1}$, 

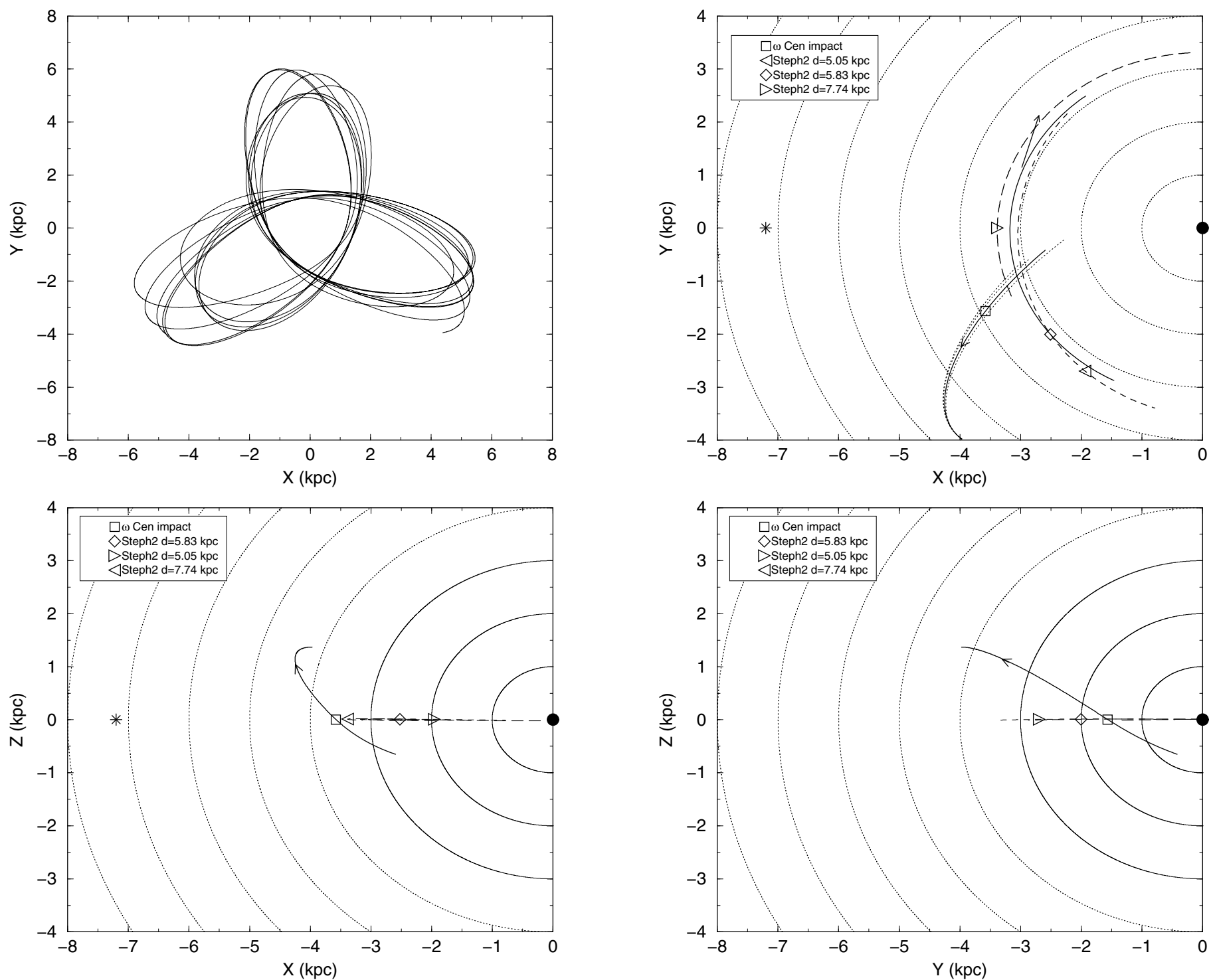

Fig. 3. Top-left panel: galactocentric $X Y$-plane projection of the $\omega$ Centauri orbit over the past 2 Gyr. Top-right: the past 30 Myr orbit of $\omega$ Centauri (solid line) for $R_{\mathrm{GC}}=7.2 \mathrm{kpc}$. Additional neighbouring orbits (dotted) are, from bottom to top: JHB96 (-10\%), JHB96 (+10\%), FSC96. The impact site on the disk is shown by the empty square. Arrows indicate orbit direction. Orbits of Stephenson 2 for the assumed distance from the Sun (and uncertainties) are shown. The corresponding $X Z$ and $Y Z$ projections are in the bottom panels. Empty symbols over the Stephenson 2 orbits indicate its possible positions $24 \mathrm{Myr}$ ago. The Sun at its present position (asterisk) and the Galactic Centre (filled circle) are shown.

$V=-44.2 \pm 8.2 \mathrm{~km} \mathrm{~s}^{-1}$, and $W=-1.3 \pm 13.0 \mathrm{~km} \mathrm{~s}^{-1}$. Alternatively, we also computed orbits with $R_{\mathrm{GC}}=7.6 \mathrm{kpc}$, which was the value obtained by Eisenhauer et al. (2005). By means of the statistical parallax of central stars, it should be noted that Trippe et al. (2008) found $R_{\mathrm{GC}}=8.07 \pm 0.32 \mathrm{kpc}$, while Ghez et al. (2008), with the orbit of one star close to the black hole, found $R_{\mathrm{GC}}=8.0 \pm 0.6 \mathrm{kpc}$ or $R_{\mathrm{GC}}=8.4 \pm 0.4 \mathrm{kpc}$, under different assumptions. Cluster distances are heliocentric, and therefore do not depend on $R_{\mathrm{GC}}$; on the other hand, the value of $R_{\mathrm{GC}}$ has some effect on the potentials, and can thus, affect the orbit computation. Since the difference between the adopted value of $R_{\mathrm{GC}}$ and the more recent measurements is insignificant, the value of $R_{\mathrm{GC}}$ should not influence significantly the present results.

Based on the rotation curves of Brand \& Blitz (1993) and Russeil (2003), and an estimate with the galaxy mass model described above (Mirabel et al. 2001), we derived the orbital velocity of Stephenson 2 to be $V_{\mathrm{c}}=214 \pm 4 \mathrm{~km} \mathrm{~s}^{-1}$. The nearly flat Galactic rotation curve at the Stephenson 2 position allows us to adopt this circular velocity also for the orbits corresponding to distance uncertainties (Sect. 1). The orbit of $\omega$ Centauri, computed back over $2 \mathrm{Gyr}$, is comparable to that derived by Mizutani et al. (2003), in particular its Rosette pattern, which is projected on the $X Y$ plane (Fig. 3). The simulation indicates that $\omega$ Centauri collided with the disk as recently as $24 \pm 2 \mathrm{Myr}$ ago. This is so short a time that fossil remains of this event may nowadays be detectable in the disk.

Figure 3 (top-left panel) shows the Galactic $X Y$-plane projection of the orbital motion of $\omega$ Centauri during the past 2 Gyr. In the remaining panels, we focus on the past $30 \mathrm{Myr}$ of the motion of $\omega$ Centauri and Stephenson 2. For Stephenson 2, we consider the different orbits resulting from the adopted distance from the Sun and their corresponding uncertainties (Sect. 1). It is interesting that the orbit of Stephenson 2 passes close to the impact site of $\omega$ Centauri at a comparable time, within the uncertainties (see below). Since Stephenson 2 and 

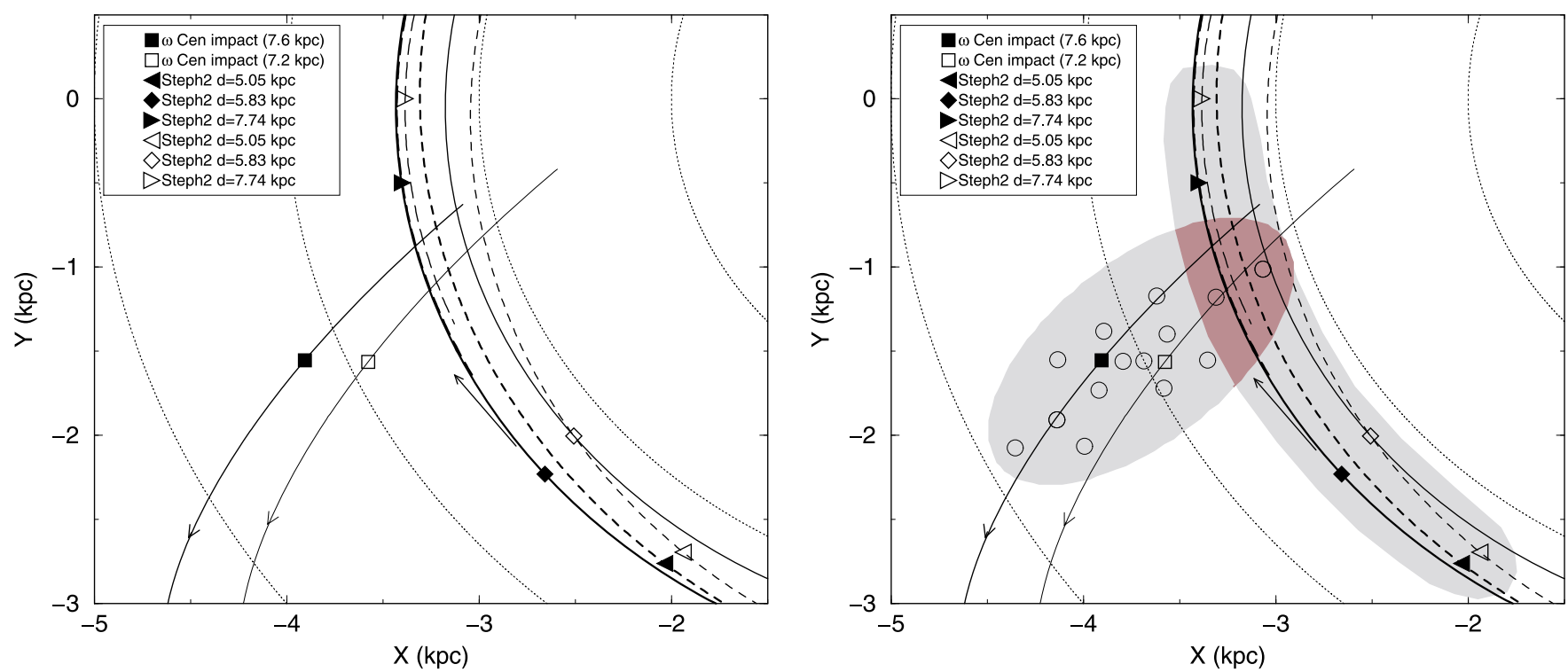

Fig. 4. Close-up of the impact site. Left panel: orbits computed with $R_{\mathrm{GC}}=7.2 \mathrm{kpc}$ (empty symbols) and $R_{\mathrm{GC}}=7.6 \mathrm{kpc}$ (filled symbols). Right: same as left panel but including error distribution for the $\omega$ Centauri impact site and Stephenson 2 proto-cluster position. A random selection of impact sites (open circles) is shown within the $\omega$ Centauri error ellipsoid.

BDSB 122 have almost the same position (within the uncertainties), the same conclusions hold for the latter cluster. The $X Z$ and $Y Z$-plane projections (bottom panels) indicate that $\omega$ Centauri emerged at $\approx 45^{\circ}$ from the plane to its present position.

To probe orbital uncertainties owing to the adopted potential, we also employed the potential model of Flynn et al. (1996) - hereafter FSC96 - and tested consequences of variations of $\pm 10 \%$ in the input parameters of JHB96. The results are shown in Fig. 3 (top-right panel), from which we conclude that orbit variations due to the adopted potential are much smaller than our error ellipsoid (Fig. 4, right panel).

Close-ups of the $\omega$ Centauri impact site and the back-traced positions of Stephenson 2 are shown in Fig. 4 (left panel) for a Sun's distance from the Galactic centre of both $7.2 \mathrm{kpc}$ and $7.6 \mathrm{kpc}$. It is clear that the value of $7.6 \mathrm{kpc}$ does not significantly alter the orbit of the encounter. The right panel shows the error ellipsoid of several impact site simulations computed by varying initial conditions according to the errors in the different relevant input quantities. The ellipsoid reflects variations implied by velocity uncertainties in the PM, radial velocity and present position of $\omega$ Centauri along the line of sight in the $(U, V, W)$ velocities. The impact obtained with a Galactocentric distance $R_{\mathrm{GC}}=7.6 \mathrm{kpc}$ is also shown. The disk-orbit of Stephenson 2 crosses the $\omega$ Centauri ellipsoid error distribution. The range in impact site to proto-cluster separations contains distances smaller than $\approx 1 \mathrm{kpc}$, with an average separation of $\sim 500 \mathrm{pc}$ (intersection area in Fig. 4, right panel). Larger separations would require prohibitive expansion velocities, despite the fact that we are dealing with an encounter in a denser, central part of the disk, while in NGC 4559, the event was external.

For the GC-induced formation hypothesis to be valid, the timescales associated with the onset of star formation (after impact), duration of star formation and the cluster age, should be compatible with the disk-crossing age. Following Vande Putte \& Cropper (2009), the first timescale in not well known, ranging from virtually instantaneous, i.e. negligible compared to the cluster age, to $15 \mathrm{Myr}$ (Lépine \& Duvert 1994) and $30 \mathrm{Myr}$ (Wallin et al. 1996). The star formation timescale may be short, $\approx 2 \times 10^{5} \mathrm{yr}$, as suggested by McKee $\&$ Tan (2002) for stars more massive than $8 M_{\odot}$. Given that the ages of Stephenson 2 and BDSB 122 are within the ranges of 12-17 Myr and 7-12 Myr, respectively, $\omega$ Centauri, which crossed the disk $\approx 24$ Myr ago, may have triggered their formation only if the star formation started during a time period of less than $\approx 15 \mathrm{Myr}$, which is within the accepted range. In the case of NGC 4559, these combined timescales are less than $\sim 30 \mathrm{Myr}$ (Soria et al. 2005).

The above clues suggest that the most recent crossing of $\omega$ Centauri through the disk occurred close to the sites where two massive OCs were formed. Both Stephenson 2 and BDSB 122 are younger than the age of the impact, and the differences in age of a few Myr are consistent with the shock propagation and subsequent star formation. The overall evidence gathered in the present analysis supports $\omega$ Centauri being the origin of this localised star formation in the Galaxy, which harbours two of the more massive known OCs.

This work suggests a scenario where the disk passage of GCs can generate OCs, massive ones in particular, as indicated by the orbit of $\omega$ Centauri and its impact site. As a consequence, OC formation is not induced entirely by the spiral density wave mechanism in spiral arms.

\section{Summary and conclusions}

Globular clusters orbiting the bulge and halo of the Galaxy cross the disk on average once every $1 \mathrm{Myr}$, and these events are expected to produce significant physical effects on the disk. For instance, the impact of a GC passing through the disk can trigger star formation either by the accumulation of gas clouds around the impact site or by the production of an expanding mechanical wave. Time delays are expected in both cases because of the collapse and fragmentation of molecular clouds before star formation. This phenomenon was observed in the galaxy NGC 4559 (e.g., SCP05). If this mechanism operates frequently in the Galaxy, the most massive GC $\omega$ Centauri can be assumed to be an ideal projectile for analysing the state of its last impact site in the disk. 
Since $\omega$ Centauri collided with the Galactic disk $\approx 24 \mathrm{Myr}$ ago, a major star-forming event appears to have occurred close $(\$ 1 \mathrm{kpc})$ to the impact locus that generated two of the most massive young OCs known in the Galaxy, BDSB 122 and Stephenson 2. We suggest a connection between these events that is similar to that between the impact and shock observed in NGC 4559 (SCP05). We use a model of the Galactic potential to integrate the orbit of $\omega$ Centauri. As shown in Fig. 4, when the uncertainties in space velocity, distances, and potential are considered, the error distributions of the $\omega$ Centauri impact site and the birth-site of Stephenson 2 overlap. This overlap suggests a scenario where the disk passage and formation of the pair of OCs may be physically connected. Alternatively, the time coincidence may have occurred within a separation $\lesssim 1 \mathrm{kpc}$. In such a case, the expanding bubble scenario such as that in NGC 4559 would apply. The latter case is more probable, since two clusters have been formed.

Levy (2000) performed 2D hydrodynamic simulations to study the impact of GCs on the Galactic disk in the presence of available gas. They found that the moving GC causes a shock in the gas that propagates through the disk on a kpc scale, thus producing star formation. Vande Putte \& Cropper (2009) simulated in detail the effects of GC impacts on the disk, basically confirming the results of Wallin et al. (1996) and Levy (2000), even in the absence of gas at the impact site. They found a concentration of disk material compressed to a scale of $\sim 10 \mathrm{pc}$, which may subsequently attract gas leading to star formation. The compression increases with the GC mass.

At this point, an interesting question arises. For a rate of $\sim 1$ GC impact per Myr, a high probability is expected of one GC impact occurring within 1-2 kpc of any location within the inner Galaxy in about $10 \mathrm{Myr}$. However, the star-formation efficiency of these events appears to be low, according to Vande Putte \& Cropper (2009), who found that of the 54 GCs with accurate proper motions studied by them, only three appear to be associated with young OCs. It is possible that conditions such as GC mass and impact site properties, and the availability of molecular gas, temperature and density, constrain the starformation efficiency.

Evidence drawn from the present work suggests that GCs can be progenitors of massive OCs. We have focused in particular on $\omega$ Centauri. Density-wave shocks may not be the only cause of the formation of the more massive OCs, which is a possibility to be further explored, both theoretically and observationally.

Acknowledgements. We thank the anonymous referee for suggestions. We acknowledge partial support from CNPq (Brazil).

\section{References}

Allen, C., Moreno, E., \& Pichardo, B. 2008, ApJ, 674, 237

Bekki, K., \& Freeman, K. C. 2003, MNRAS, 346, L11

Bica, E., Santos, Jr., J. F. C., \& Alloin, D. 1990, A\&A, 235, 103

Bica, E., Dutra, C. M., Soares, J., \& Barbuy, B. 2003, A\&A, 400, 533

Bica, E., Bonatto, C., Barbuy, B., \& Ortolani, S. 2006, A\&A, 450, 105

Bica, E., Bonatto, C., Ortolani, S., \& Barbuy, B. 2007, A\&A, 472, 483

Brand, J., \& Blitz, L. 1993, A\&A, 275, 67

Davies, B., Figer, D. F., Kudritzki, R.-P., et al. 2007, ApJ, 671, 781

Dias, W. S., Alessi, B. S., Moitinho, A., \& Lépine, J. R. D. 2002, A\&A, 389, 871 Dinescu, D. I., Girard, T. M., van Altena, W. F., \& López, C. E. 2003, AJ, 125, 1373

Drimmel, R., \& Spergel, D. N. 2001, ApJ, 556, 181

Dutra, C. M., Bica, E., Soares, J., \& Barbuy, B. 2003, A\&A, 404, 223

Eisenhauer, F., Genzel, R., Alexander, T., et al. 2005, ApJ, 628, 246

Elmegreen, B. G., \& Lada, C. J. 1977, ApJ, 214, 725

Elmegreen, B. G., Efremov, Y. N., \& Larsen, S. 2000, ApJ, 535, 748

Figer, D. F., MacKenty, J. W., Robberto, M., et al. 2006, ApJ, 643, 1166

Flynn, C., Sommer-Larsen, J., \& Christensen, P. R. 1996, MNRAS, 281, 1027

Ghez, A. M., Salim, S., Weinberg, N. N., et al. 2008, ApJ, 689, 1044

Hanson, M. M., \& Bubnick, B. F. 2008, PASP, 120, 150

Hernquist, L. 1990, ApJ, 356, 359

Johnston, K. V., Hernquist, L., \& Bolte, M. 1996, ApJ, 465, 278

Larsen, S. S., Efremov, Y. N., Elmegreen, B. G., et al. 2002, ApJ, 567, 896

Lépine, J. R. D., \& Duvert, G. 1994, A\&A, 286, 60

Levy, V. V. 2000, A\&AT, 18, 621

McKee, C. F., \& Tan, J. C. 2002, Nature, 416, 59

Mirabel, I. F., Dhawan, V., Mignani, R. P., \& Rodrigues, I. 2001, Nature, 413, 139

Miyamoto, M., \& Nagai, R. 1975, PASJ, 27, 533

Mizutani, A., Chiba, M., \& Sakamoto, T. 2003, ApJ, 589, L89

Momany, Y., Zaggia, S., Gilmore, G., et al. 2006, A\&A, 451, 515

Nakaya, H., Watanabe, M., Ando, M., Nagata, T., \& Sato, S. A. 2001, AJ, 122, 876

Ortolani, S., Bica, E., Barbuy, B., \& Momany, Y. 2002, A\&A, 390, 931

Ortolani, S., Bonatto, C., Bica, E., Momany, Y., \& Barbuy, B. 2008, NewA, 13, 508

Paczyński, B. 1998, ApJ, 494, L45

Rees, R. F., \& Cudworth, K. M. 2003, BAAS, 35, 1219

Russeil, D. 2003, A\&A, 397, 133

Soares, J., Bica, E., Ahumada, A. V., \& Clariá, J. J. 2008, A\&A, 478, 419

Soria, R., Cropper, M., \& Pakull, M. 2004, RMxAC, 20, 57

Soria, R., Cropper, M., Pakull, M., Mushotzky, R., \& Wu, K. 2005, MNRAS, 356,12

Stephenson, C. B. 1990, AJ, 99, 1867

Struck-Marcell, C., \& Higdon, J. L. 1993, ApJ, 411, 108

Struck-Marcell, C., Appleton, P. N., Borne, K. D., \& Lucas, R. A. 1996, AJ, 112, 1868

Trippe, S., Gillessen, S., Gerhard, O. E., et al. 2008, A\&A, 492, 419

Vande Putte, D., \& Cropper, M. 2009, MNRAS, 392, 113

Vlemmings, W. H. T., Cordes, J. M., \& Chatterjee, S. 2004, ApJ, 610, 402

Wallin, J. F., Higdon, J. L., \& Staveley-Smith, L. 1996, AJ, 459, 555

Whitworth, A. P., Bhattal, A. S., Chapman, S. J., Disney, M. J., \& Turner, J. A. 1994, A\&A, 290, 421 